Wright State University

CORE Scholar

9-1-1997

\title{
High Quality Interfaces in GaAs-Alas Quantum Wells Determined from High Resolution Photoluminescence
}

D. C. Reynolds

David C. Look

Wright State University - Main Campus, david.look@wright.edu

B. Jogai

R. Kaspi

K. R. Evans

See next page for additional authors

Follow this and additional works at: https://corescholar.libraries.wright.edu/physics

Part of the Physics Commons

\section{Repository Citation}

Reynolds, D. C., Look, D. C., Jogai, B., Kaspi, R., Evans, K. R., \& Estes, M. (1997). High Quality Interfaces in GaAs-Alas Quantum Wells Determined from High Resolution Photoluminescence. Journal of Vacuum Science \& Technology B, 15 (5), 1703-1706.

https://corescholar.libraries.wright.edu/physics/172

This Article is brought to you for free and open access by the Physics at CORE Scholar. It has been accepted for inclusion in Physics Faculty Publications by an authorized administrator of CORE Scholar. For more information, please contact library-corescholar@wright.edu. 
Authors

D. C. Reynolds, David C. Look, B. Jogai, R. Kaspi, K. R. Evans, and M. Estes

This article is available at CORE Scholar: https://corescholar.libraries.wright.edu/physics/172 


\title{
High quality interfaces in GaAs-AIAs quantum wells determined from high resolution photoluminescence
}

\author{
D. C. Reynolds, D. C. Look, ${ }^{\text {a) }}$ and B. Jogai \\ University Research Center, Wright State University, Dayton, Ohio 45435 \\ R. Kaspi, K. R. Evans, and M. Estes \\ Avionics Directorate, Wright Laboratory, WL/AADP, Wright-Patterson Air Force Base, Ohio 45433
}

(Received 24 April 1997; accepted 25 July 1997)

\begin{abstract}
High resolution photoluminescence (PL) measurements performed on several GaAs-AlAs quantum well structures revealed sharp excitonic transitions separated in energies corresponding to roughly half-monolayer fluctuations in well size. The narrow linewidths correlate with interface island structure whose lateral extent is either much larger or much smaller than the exciton diameter. The half-monolayer separation results from a sharply peaked PL intensity response occurring around those areas of the laterally nonuniform interface which have roughly $50 \%$ island coverage, with the average island size much smaller than the exciton diameter, about $225 \AA$. (C) 1997 American Vacuum Society. [S0734-211X(97)04505-8]
\end{abstract}

\section{INTRODUCTION}

The quality of semiconductor heterointerfaces has been investigated many times over the past several years. The materials most commonly used to produce the heterostructures are GaAs and AlGaAs. To gain a better understanding of the interface quality, electronic ${ }^{1-3}$ and optical ${ }^{4,5}$ properties associated with the interfaces have been studied. High, twodimensional electron gas (2DEG) mobilities have been observed for GaAs-AlGaAs heterojunctions in which the GaAs layer is grown before the doped AlGaAs layer. ${ }^{1,2}$ Interfaces grown in reverse order show lower mobilities. ${ }^{3}$ Weisbuch et al. ${ }^{4}$ studied the optical emission from GaAs-AlGaAs multiple quantum well structures; they found that thickness fluctuations (islands) were present in each well at the interface. Their analysis supported a model of an islandlike interface with a height of 1 monolayer (ML) and a lateral island size of approximately $300 \AA$. A similar model was proposed by Goldstein et al.,,$^{5}$ also on multi-quantum well structures. Later studies ${ }^{6,7}$ showed fine structure features on both the free exciton transitions and donor bound exciton transitions associated with GaAs-AlGaAs multi-quantum well structures. The observed fine structure was explained in terms of changes in the average well size; in this case ${ }^{6,7}$ effective well size variations of less than $1 \mathrm{ML}$ were observed.

The GaAs-AlAs heterostructure system has also received considerable attention. In related investigations the observed well size fluctuations were $\sim 1 \mathrm{ML} .{ }^{8,9}$ In the current article we report on GaAs-AlAs single quantum wells where the predominant effective well size fluctuation is $\sim 1 / 2 \mathrm{ML}$. Three different well sizes (nominally 50, 75, and $100 \AA$ ) were investigated. The observed linewidths are narrow, some as narrow as $0.6 \mathrm{meV}$, indicating very good sample quality and accompanying interface structure. The sharpness of the individual peaks indicates that most of the interface islands have sizes either much larger or much smaller than the exci-

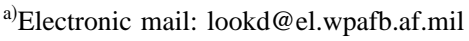

ton diameter. ${ }^{7}$ We show that varying the power density of the exciting laser has a marked influence on the emission characteristics.

\section{EXPERIMENTAL METHOD}

The structures used in these experiments were grown by solid source molecular beam epitaxy, in two different highpurity modified commercial molecular beam epitaxy (MBE) reactors, using a substrate temperature of $\sim 600{ }^{\circ} \mathrm{C}$ and incident $\mathrm{Al}$ and $\mathrm{Ga}$ fluxes in the range of $0.5-1.0 \mathrm{ML} / \mathrm{s}$. The arsenic source was dimeric $\mathrm{As}_{2}$. Some structures incorporated a small mole fraction $(y \sim 0.02)$ of indium in the barrier region for the purpose of another study. The results of the present study were found to be independent of the presence of indium. Three different nominal well sizes; 50, 75, and $100 \AA$, all with $100 \AA$ barriers, were selected for this study. Layer thicknesses were calculated based on conventional flux calibration measurements using reflection high energy electron diffraction (RHEED). The photoluminescence (PL) was excited with an $\mathrm{Ar}^{+}$ion laser. The measurements were made at $2 \mathrm{~K}$ with the sample immersed in liquid He. The spectra were analyzed with a high resolution $4 \mathrm{~m}$ spectrometer equipped with an RCA C31034A photomultiplier tube for detection.

\section{EXPERIMENTAL RESULTS}

The PL spectra observed for the 50, 75, and $100 \AA$ A quantum wells are shown in Fig. 1. We note that the separated PL peaks observed for the three different well thicknesses all correspond to approximately $1 / 2$ ML changes in well thickness. The calculated energy separations for monolayer and half monolayer fluctuations in well size for the three different thicknesses are given in Table I. The energies were calculated from an $8 \times 8 \mathrm{~K} \cdot \mathrm{P}$ formulation. The band alignment was assumed to be type I with the confinement potential taken as $\Gamma_{\mathrm{GaAs}}-\Gamma_{\mathrm{AlAs}}$. For wells that are greater than $30 \AA$ in width, with barriers equal to or greater than the well 


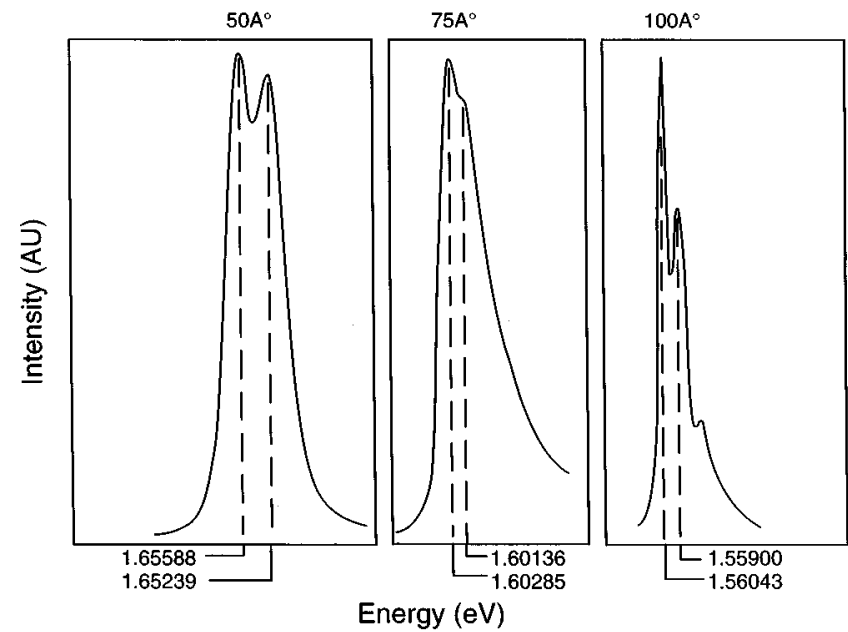

FIG. 1. Free exciton fine structure resulting from approximately $1 / 2 \mathrm{ML}$ fluctuation in well size for three different sized wells. The spectra are normalized at their respective peak values.

width, $\Gamma_{\text {AlAs }}$ rather than $X_{\text {AlAs }}$ is the appropriate choice. ${ }^{10}$ The modified Luttinger parameters were fitted to the bulk effective masses of the constituent materials. Also given in Table I are the experimentally observed values of the energy separations. A theoretical model that explains fractional monolayer variations in well size was developed by Singh et $a l^{7,11,12}$ According to the model, the interface can be represented by GaAs and AlAs-like two-dimensional (2D) islands. For simplicity we have placed all of the interface structure at the AlAs on GaAs interface. This is a reasonable assumption since the inverted interface in this system is known to be considerably rougher than the normal interface. Due to the high migration rate of $\mathrm{Ga}$ atoms, the normal interface is expected to be sharp to a monolayer. The quality of the inverted interface is controlled by the $\mathrm{Al}$ migration rate. The $\mathrm{Al}$ migration rate is expected to be much smaller than that for Ga because the the Al-As bond is stronger than the $\mathrm{Ga}-\mathrm{As}$ bond, which suggests a lower hopping rate for $\mathrm{Al}$ atoms. ${ }^{12}$ In the layer by layer growth mode, the layer coverage will be determined by the size and density of these islands. Consider the case in which AlAs growth is initiated on the previous GaAs layer. It is recognized that in the growth process it is unlikely that the shutter can be controlled so that

TABLE I. Energy of the heavy-hole exciton for the narrow well $(E)$, change in energy due to one monolayer increase in well size $(\Delta E)$, and the change in energy due to a half monolayer increase in well size $(\Delta E / 2)$. One monolayer is taken to be $2.8 \AA$.

\begin{tabular}{|c|c|c|c|c|}
\hline $\begin{array}{l}L_{z} \\
(\AA)\end{array}$ & $\begin{array}{c}E \\
(\mathrm{eV})\end{array}$ & $\begin{array}{c}\Delta E \\
(\mathrm{meV})\end{array}$ & $\begin{array}{c}\Delta E / 2 \\
(\mathrm{meV})\end{array}$ & Experiment \\
\hline $\begin{array}{l}100 \\
102.8\end{array}$ & 1.5659 & 2.4 & 1.2 & 1.4 \\
\hline $\begin{array}{l}75 \\
77.8\end{array}$ & 1.5948 & 4.7 & 2.3 & 1.7 \\
\hline $\begin{array}{l}50 \\
52.8\end{array}$ & 1.6591 & 10.0 & 5.0 & 3.5 \\
\hline
\end{tabular}

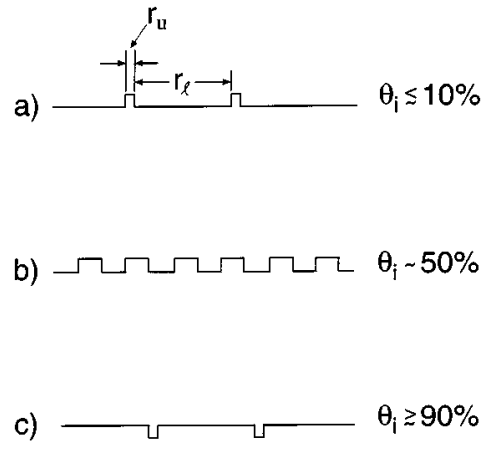

FIG. 2. Schematic representation of the microstructure of a surface growing in the layer-by-layer growth mode. $r_{u}$ and $r_{l}$ are the dimensions associated with the layer $i$ and $i-1$, where layer $i$ starts to grow on layer $i-1 . \Theta_{i}$ represents the coverage of layer $i$.

exactly integral monolayers are grown. If the Ga shutter is closed slightly after the previous GaAs layer is completed, then there will be a few, small GaAs islands of dimension $r_{u}$ and separated by $r_{l}$, sitting on top of a complete GaAs layer. After the AlAs fills in this layer, the interface will consist of these small GaAs islands interspersed among large AlAs islands. This situation, shown in Fig. 2(a), can be represented by a fractional GaAs coverage $\Theta_{i} \ll 1$. The excitons of dimension $R_{\text {ex }}\left(r_{u} \ll R_{\text {ex }} \ll r_{l}\right)$ will mainly see pure AlAs at the interface, and thus will be sharp. Next, suppose that the first AlAs layer is started after the previous GaAs layer is nearly complete $\left(\Theta_{i} \simeq 1\right)$; then the interface will consist of large GaAs islands and small AlAs islands, as represented by Fig. 2(c). In this case, the excitons will mainly see pure GaAs islands at the interface $\left(r_{l} \ll R_{\mathrm{ex}} \ll r_{u}\right)$ and again will be sharp, although separated in energy by $\Delta E\left(W_{0}\right)$ from the previous case (where $W_{0}$ is the average well width). Statistically, a typical interface can consist of these two cases and all others in between. However, at roughly $50 \%$ coverage $\left(\Theta_{i} \simeq 0.5\right)$, if the island size $r_{u}$ is such that both $r_{u}$ and $r_{l}$ are much smaller than $R_{\mathrm{ex}}$, then the exciton will see many islands of both types, and the PL line will again be narrow, ${ }^{7}$ but now separated by $\Delta E\left(W_{0}\right) / 2$ from each of the two previous cases. Thus, in this model of a $1 \mathrm{ML}$, laterally nonuniform interface, a quantum well (QW) PL spectrum could consist of one, two, or three sharp lines, separated by $\Delta E\left(W_{0}\right)$ or $\Delta E\left(W_{0}\right) / 2$, on top of a broad background due to the parts of the interface not having $\Theta_{i} \simeq 0,0.5$, or 1 . If the $\Theta_{i} \simeq 0.5$ case happened to consist of islands with $r_{u} \simeq R_{\mathrm{ex}}$, then the $\Delta E\left(W_{0}\right) / 2$ line would not be sharp, and this might explain some of the literature results which contain only $\Delta E\left(W_{0}\right)$ separations. We might also note that if the interface variations cover $2 \mathrm{ML}$, rather than one, then more than three lines can appear.

Warwick et al. ${ }^{13}$ take issue with what is termed the common literature concept that the interface will consist of perfectly smooth, 1-ML-high mesas ("islands") of large extent, say $1-10 \mu \mathrm{m}$ in width, sitting on a flat background. Indeed, if this were the case, and if both the mesa widths and the distances between mesas were larger than $R_{\mathrm{ex}}$, then the exciton spectrum would consist of only two sharp lines, sepa- 


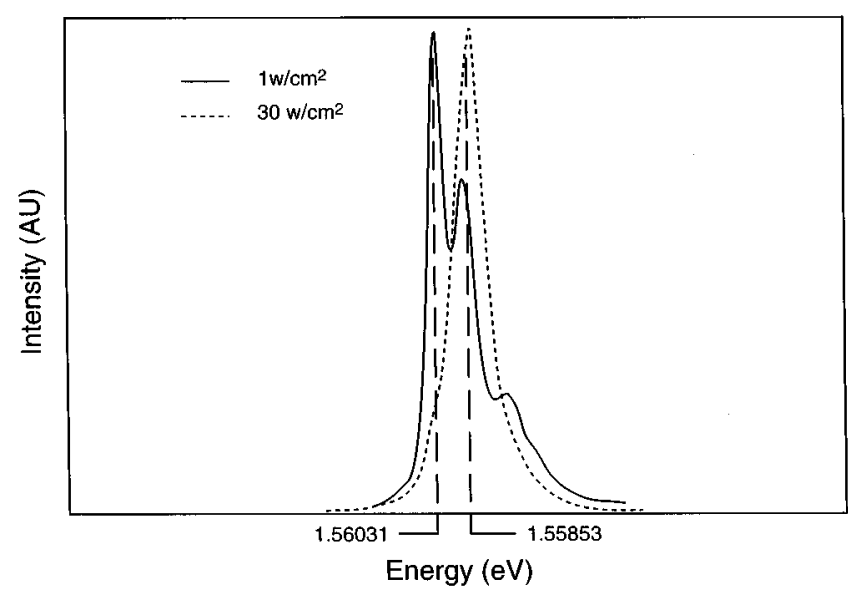

FIG. 3. Emission intensity reversal between the narrower portion and the wider portion of the $100 \AA$ quantum well, as the exciting intensity is increased from 1 to $30 \mathrm{~W} / \mathrm{cm}^{2}$. The spectra are normalized.

rated by exactly $\Delta E\left(W_{0}\right)$. Obviously, this is not generally the case (as shown, e.g., in the present results). Our model, represented by Fig. 2, recognizes that a distribution of $r_{l}$ and $r_{u}$ sizes will exist for every interface. The only constraint, as pointed out in our discussion above, is that the $r_{l}$ and $r_{u}$ cannot have strong contributions near $R_{\mathrm{ex}}$, or otherwise the PL lines would not be sharp. These considerations demonstrate the power of the exciton as a probe: the linewidth relates to the distribution of island sizes (or roughness) and the energy spacing, to the extent of coverage.

It was reported by Gammon et al. ${ }^{8}$ that the laser exciting intensity had little effect on the spectral character of the emission from the GaAs-AlAs QWs that they investigated. We find this not to be the case for the QWs currently being reported. Consider the dynamics of forming an exciton in a quantum well with narrow and wide regions. For excitation energies above the band gap, the initially formed, laserexcited level is that of a free-electron and hole with finite momenta. The free carriers quickly relax to form delocalized free excitons which are free to migrate within energetically accessible regions of the QW in which they are formed. The excitons created in the higher formation energy regions, and thus, effectively narrower well regions, are free to migrate not only within their formation regions but also can migrate downhill in energy to lower energy, effectively wider width regions. This migration process competes directly with the process of converting delocalized free excitons into localized free excitons via "trapping", at local potential minima. The localized free excitons will then radiatively recombine reflecting the environment of their localization which can be in either the narrower or wider regions of the respective wells. Considering the $100 \AA$ well, at low exciting intensities, $1 \mathrm{~W} / \mathrm{cm}^{2}$, the intensity of the narrow portion of the well exceeds that of the wider portion. As the exciting intensity increases to $\sim 30 \mathrm{~W} / \mathrm{cm}^{2}$, the intensity of the wider portion of the well exceeds that of the narrower portion as shown in Fig. 3. The intensity behavior of the $50 \AA$ well is distinctly different as seen in Fig. 4. At low exciting intensities the

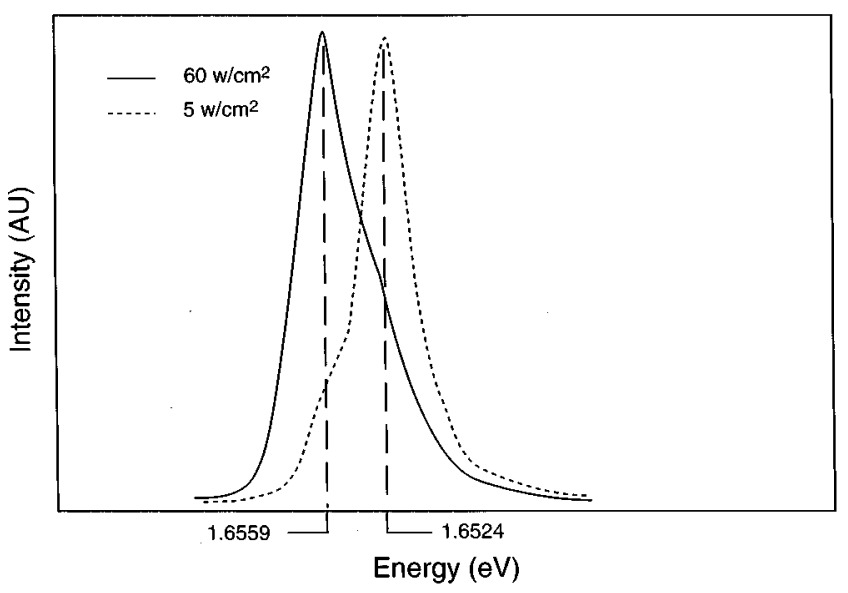

FIG. 4. Emission intensity reversal between the narrower portion and the wider portion of the $50 \AA$ quantum well, as the exciting intensity is increased from 5 to $60 \mathrm{~W} / \mathrm{cm}^{2}$. It is noted that the intensity reversal for this well is opposite to what was observed for the $100 \AA$ quantum well. The spectra are normalized.

emission intensity of the wider portion of the well exceeds that of the narrower portion, whereas the reverse becomes true as the exciting intensity increases. The intensity distributions as a function of exciting intensities reflect the localized positions of the excitons as they radiatively recombine. The details of the exciton dynamics are not understood, although they must involve energy as well as fractional coverage considerations. However, it is important to note that in contrast to our observations, the spectra discussed in Ref. 8 were not influenced by excitation intensity. These phenomena require further investigation.

In summary, we have shown that the interfaces contained in the present QWs differ in two important aspects with those previously reported. The linewidths we report are very narrow and correlate with an effective 1/2 ML change in well thickness. Previously only ML and larger fluctuations were observed. Also, in contrast to previous results, PL spectra were markedly influenced by the power density of the exciting laser.

\section{ACKNOWLEDGMENTS}

The authors would like to thank C. Huang and C. W. Litton for technical support. The work of D.C.R., D.C.L., and B.J. was performed at Wright Laboratory, Solid State Electronics Directorate (WL/EL), Wright Patterson Air Force Base under USAF Contract No. F33615-95-C-1619. This work was partially supported by Air Force Office of Scientific Research (AFOSR).

${ }^{1}$ H. L. Stormer, A. Pinczuk, A. C. Gossard, and W. Weigmann, Appl. Phys. Lett. 38, 692 (1981).

${ }^{2}$ L. C. Witkowski, T. J. Drummond, C. M. Stanchak, and H. Morkoc, Appl. Phys. Lett. 37, 1033 (1980).

${ }^{3}$ T. J. Drummond, H. Morkoc, S. L. Su, R. Fischer, and A. Y. Cho, Electron. Lett. 17, 870 (1981).

${ }^{4}$ C. Weisbuch, R. Dingle, A. C. Gossard, and W. Weigmann, in Gallium Arsenide and Related Compounds, edited by H. W. Thim, Inst. Phys. Conf. Ser. 56 (IOP, Bristol, 1981), Chap. 9, p. 711.

${ }^{5}$ L. Goldstein, Y. Horokoshi, S. Tarucha, and H. Akamoto, Jpn. J. Appl. Phys., Part 1 22, 1489 (1983). 
${ }^{6}$ D. C. Reynolds, K. K. Bajaj, C. W. Litton, P. W. Yu, Jasprit Singh, W. T. Masselink, R. Fischer, and H. Morkoc, Appl. Phys. Lett. 46, 51 (1985).

${ }^{7}$ Jasprit Singh, K. K. Bajaj, D. C. Reynolds, C. W. Litton, P. W. Yu, W. T. Masselink, R. Fischer, and H. Morkoc, J. Vac. Sci. Technol. B 3, 1061 (1985).

${ }^{8}$ D. Gammon, B. V. Shanabrook, and D. S. Katzer, Semiconductor Interfaces and Microstructures, edited by Z. C. Feng (World Scientific, Singapore, 1992), Chap. 6, p. 149, and references therein.
${ }^{9}$ M. Proctor, G. Oelgart, H. Rhan, and F. K. Reinhart, Appl. Phys. Lett. 64, 3154 (1994).

${ }^{10}$ J. Ihm, Appl. Phys. Lett. 50, 10681 (1987).

${ }^{11}$ Jasprit Singh, K. K. Bajaj, and S. Chaudhuri, Appl. Phys. Lett. 44, 805 (1984).

${ }^{12}$ Jasprit Singh and K. K. Bajaj, J. Vac. Sci. Technol. B 3, 520 (1985).

${ }^{13}$ C. A. Warwick, W. Y. Jan, A. Ourmazd, and T. D. Harris, Appl. Phys. Lett. 56, 2666 (1990). 